\title{
On the Relationship between Statistical and Phenomenological Models of the Thermodynamic Systems
}

\author{
Igor Samkhan \\ Department of Heat Engines, Yaroslavl State Technical University, City of Yaroslavl, Russia \\ Email: eneres1@gmail.com
}

Received May 3, 2013; revised June 6, 2013; accepted July 3, 2013

Copyright (c) 2013 Igor Samkhan. This is an open access article distributed under the Creative Commons Attribution License, which permits unrestricted use, distribution, and reproduction in any medium, provided the original work is properly cited.

\begin{abstract}
The paper deals with the performing of a critical analysis of the problems arising in matching the classical models of the statistical and phenomenological thermodynamics. The performed analysis shows that some concepts of the statistical and phenomenological methods of describing the classical systems do not quite correlate with each other. Particularly, in these methods various caloric ideal gas equations of state are employed, while the possibility existing in the thermodynamic cyclic processes to obtain the same distributions both due to a change of the particle concentration and owing to a change of temperature is not allowed for in the statistical methods. The above-mentioned difference of the equations of state is cleared away when using in the statistical functions corresponding to the canonical Gibbs equations instead of the Planck's constant a new scale factor that depends on the parameters of a system and coincides with the Planck's constant in going of the system to the degenerate state. Under such an approach, the statistical entropy is transformed into one of the forms of heat capacity. In its turn, the agreement of the methods under consideration in the question as to the dependence of the molecular distributions on the concentration of particles, apparently, will call for further refinement of the physical model of ideal gas and the techniques for its statistical description.
\end{abstract}

Keywords: Thermodynamics; Classical Systems; Description Models; Statistical Functions; Phase Space; Probability Distribution; Particle Concentration

\section{Introduction}

At present, two methods are used to describe the thermodynamic systems being an assemblage of randomly moving microparticles [1]. In one of them represented by the phenomenological classical thermodynamics, such systems are considered as a single whole without regard for their internal structure, with the properties of the system being characterized by several macroscopic parameters.

In another method being referred to as statistical thermodynamics or statistical physics, the models of the system's internal structure allowing for changes in energy and coordinates of individual molecules in their interaction between themselves and the environment are treated. The results of such interaction are usually represented as the probability of distribution of the molecules according to energies, momenta, coordinates and the like microparameters of a system. In this case the macroscopic parameters of the system are determined by the statistical methods as mean factors of the molecular ensemble, which can appear with the highest degree of probability during a long period of observation.

It is generally suggested that both methods complementing each other agree nicely with one another, thus demonstrating the unity of the thermodynamic approach and the reliability of well-known thermodynamic concepts.

However, such an agreement cannot be yet accepted as sufficiently complete because of the existence in the statistical thermodynamics of a number of the concepts, which do not quite correlate with the principles of the phenomenological thermodynamics and the existing practice.

Specifically, various caloric ideal gas equations of state [2] are employed in these techniques, besides the assumption of the statistical method about the independence of the classical distributions by velocities and momenta from the concentration of particles is hard to fit to possible change of the distributions in the thermodynamic cycles.

The existing difficulties of the statistical thermodynamics for classical systems are often not stated in an 
explicit form or are justified to some extent with references to a partial agreement of the theory with the experimental measurements or to a possibility of refinement of the classical approaches in the quantum statistics, for example, in the Bose-Einstein or Fermi-Dirac statistics. However, the experimental investigations do not cover every possible case, and the physical models of the quantum and classical statistics do not correlate very well with each other. In particular, in the quantum statistics as distinct from the classical ones the identical particles are deemed indistinguishable, and the molecules can be considered in energetically excited states which are not taken into consideration in the classical systems, e.g. in the translational motion of the ideal gas molecules [3].

The presence of similar problems in the agreement and substantiation of the thermodynamic techniques makes necessary a search for further ways of their development. One of the steps in this direction pertaining to the refinement of the entropy behavior in the adiabatic processes of the open thermodynamic systems has been examined comparatively recently in paper [4].

In the present paper, some problems and possible ways associated with a more comprehensive agreement of various branches of the thermodynamics are treated from the standpoint of the statistical method.

\section{Dependence of the Statistical Functions of the Thermodynamic Systems on the Scale Factor}

One of the problems hampering the matching of the phenomenological and statistical models of the thermodynamics is the lack in the former case of such notion as the absolute entropy $S$, whereas it forms the basis of the statistical method.

This circumstance seems at first sight to be of small importance because the statistical entropy is usually determined only to an accuracy of the arbitrary constant or otherwise the scale factor $\alpha$, for example, using the formula

$$
S=k N \ln \frac{V \mathrm{e}^{5 / 2}}{N}\left(\frac{2 \pi m k T}{a^{2}}\right)^{3 / 2}
$$

where $N$ is the number of particles, $V$ is the volume, $T$ is the temperature of the thermodynamic system, $m$ is the mass of molecules, $k$ and $\mathrm{e}$ are the Boltzmann constant and the base of natural logarithm, respectively [3].

In so doing, the effect of this constant when calculating the entropy difference can be eliminated by assuming that the value of $\alpha$ does not depend on the parameters of the system and is equal for various systems.

However, such an assumption leads to additional difficulties. In particular, it is generally supposed that the Planck's constant raised to the third power can be chosen as such constant for classical (molecular) systems

$$
\alpha^{3}=h^{3}=(\lambda p)^{3}=\left(6.6 \times 10^{-34}, \mathrm{Js}\right)^{3} .
$$

This coefficient is typically used for the description of the quantum phenomena of the microworld and, particularly, for the relations between the quantum energy $e$ and the oscillation frequency of electromagnetic radiation $v$

$$
e=h v \text {, }
$$

or between the de Broglie wavelength $\lambda$ and the momentum of particle $p$

$$
\lambda=h / p
$$

In so doing, the application of the Planck's constant to the description of classical systems seems logically attractive for developing a general thermodynamic conception.

At the same time such an approach does nevertheless not quite conform to the physical nature of the classical systems and to the relationships between their parameters obtained using the molecular-kinetic theory.

Thus, it is common knowledge that the degeneracy temperature $T_{g}$ characterizing the possibility of the quantum effects appearing in the classical systems

$$
T_{g}=\frac{h^{2}}{2 k m}\left(\frac{3 n}{8 \pi}\right)^{2 / 3},
$$

is for the majority of ideal gases very small; it depends on the concentration $n$ and the mass $m$ of particles and makes up, e.g. for helium at standard conditions, $T_{g}=0.05 \mathrm{~K}$, decreasing for the gases with greater molecular mass [5].

Moreover, such choice of the scale factor entails the employment in the phenomenological thermodynamics and classical statistics of various caloric equations of state, which do not quite correlate with each other. In particular, the caloric ideal gas equation of state

$$
T C_{p}=U+P V=\left(C_{v}+R\right) T
$$

incorporates only macroscopic parameters of the system, such as the internal energy $U$, the volume $V$, the pressure $P$ and the heat capacity $C_{p}$ at $P=$ const or the heat capacity $C_{v}$ at $V=$ const, and the gas constant $R$.

Whereas the canonical Gibbs equation of state

$$
T S=U-F=U+P V+\mu N
$$

involves additionally a number of statistical parameters, including the free energy of the system

$$
F=-P V-\mu N
$$

and its chemical potential 


$$
\mu=k T \ln \frac{V}{N}\left(\frac{m k T}{2 \pi \hbar^{2}}\right)^{3 / 2}
$$

for finding of which, apart from the mass $m$ and the number $N$ of the particles, such parameters of the internal structure of the system as the coordinates of the particles $x, y, z$, the projection of their momenta $p_{x,} p_{y}, p_{z}$ on the axes of a rectangular coordinate system as well as the elementary volumes

$d g\left(d g=d x d y d z d p_{x} d p_{y} d p_{z}\right)$ of the imaginary multidimensional phase space, are taken into account too [1].

It may appear thereby that canonical Equation (7) as compared with Equation (6) contains far more complete information about the system, incorporating also the data on the internal structure of the system and its possible microstates.

However, this opinion is not quite justified because with allowance made for the formulas (6) and $C_{p}=(5 / 2) k N$ the canonical Equation (7) can be represented as Equation (10)

$$
T S=T C_{p}+\mu N,
$$

in which the statistical summand $\mu N$ should be taken equal to zero or to another constant magnitude. This circumstance emerges from the thermodynamic determination of change in the entropy of the system, for example, in an isobaric process

$$
\Delta S=C_{p} \ln \left(T_{2} / T_{1}\right),
$$

in which the summand $\mu N$ is not taken into consideration at all, and from the necessity of an identical notion of the entropy in both methods of the thermodynamics.

One way to surmount this difficulty may lie with the assumption, which appears not to be considered previously, about the advisability of using in the statistical description of the classical systems the variable scale factor depending on the parameters of the system.

To realize such an approach let us represent the scale factor $a^{3}=h_{c}^{3}$ for classical systems as Equation (12)

$$
h_{c}=\lambda_{c} p
$$

determining the dependence of this factor on the average statistical parameters of the system: the length $\lambda_{c}$ of the elementary volume $\lambda_{c}^{3}=V / N$ falling at one molecule of ideal gas, and the momentum of particles $p=m v$.

Equation (12) is similar in form to the known de Broglie relationship

$$
h=\lambda p,
$$

correlating the Planck's constant $h$, the de Broglie wavelength $\lambda$ and the momentum of particle $p$.

Both above formulas coincide with each other when the value of the scale factor becomes equal to the Planck's constant $h_{c}=h$, what from a physical point of view signifies the transformation of the classical gas into the degenerate state in which quantum effects manifest themselves.

With due regard for Equation (12) the scale factor for the classical systems having a three-dimensional motion of gas molecules may be represented as Equation (14)

$$
a^{3}=h_{c}^{3}=\frac{V}{N}(2 \pi m k T)^{3 / 2},
$$

in which the multiplier $p_{c}^{3}=(2 \pi m k T)^{3 / 2}$ corresponds to the herein accepted projection of the average statistical momentum of particles of the system on the axes of a rectangular coordinate system

$$
p_{c}=(2 \pi m k T)^{1 / 2} \text {. }
$$

In so doing, the accepted value $p_{c}$ coincides with the magnitude of the known statistical integral in the Maxwell's and Gibbs distributions

$$
p_{c}=\int_{-\infty}^{\infty} \mathrm{e}^{-\frac{p_{i}^{2}}{2 m k T}} \mathrm{~d} p_{x}
$$

and differs from the known root-mean-square projection of such momentum

$$
p_{m}=(3 m k T)^{1 / 2}
$$

only by a near-one numerical coefficient.

Equation (14) derived here is virtually analogous to a known criterion of the statistical physics

$$
\gamma=\frac{V}{N}\left(\frac{2 \pi m k T}{h^{2}}\right)^{3 / 2},
$$

generally applied along with $T_{g}$ to the assessment of the degree of manifestation of the quantum effects in the ideal gas, i.e. to the determination of the question as to a possibility of considering the behavior of gas from a classical $(\gamma \gg 1)$, quantum $(\gamma \ll 1)$ or intermediate $(\gamma \approx 1)$ standpoint [6].

However, contrary to Equation (18) in which the Planck's constant $h$ is used as scale factor, Equation (14) has another physical meaning, determining the dependence of the scale factor on the parameters of the classical systems, both of these expressions coinciding at $a=h$.

The employment of the variable scale factor enables a number of the known statistical functions of the classical systems to be interpreted in a new fashion. In particular, the known statistical functions of the monoatomic ideal gas, when substituting in them for the Planck's constant the scale factor according to Equation (14), are rearranged to the form:

$$
\mu=k T \ln \frac{V}{N}\left(\frac{2 \pi m k T}{a^{2}}\right)^{3 / 2}=0
$$




$$
\begin{gathered}
F=-k T N \ln \frac{V e}{N}\left(\frac{2 \pi m k T}{a^{2}}\right)^{3 / 2}=k T N=p V \\
S=k N \ln \frac{V e^{5 / 2}}{N}\left(\frac{2 \pi m k T}{a^{2}}\right)^{3 / 2}=\frac{5}{2} k N=C_{p},
\end{gathered}
$$

showing that in this event the chemical potential of the system is equal to zero $(\mu=0)$, the free energy of the system is equal to $F=p V$, while the system entropy $S$ may be identified with the heat capacity at a constant pressure $S=C_{p}$.

In this interpretation as well as in [2,4] such parameters as the heat capacity $C_{p}=C_{v}+R$, the heat capacity $C_{v}$ at a constant volume and the gas constant $R$ are considered as the functions depending not only on the temperature, but also on the specific volume of the system.

In that event the known canonical Equation (7) of ideal gas state being a consequence of the statistical method is rearranged to a similar Equation (6) of the classical thermodynamics, what eliminates the discrepancies existing currently between them.

Moreover, Equation (6) of the ideal gas state can be obtained by a statistical method without using the conventional concepts about the phase space of thermodynamic systems and their elementary cells (the scale factor).
In this case, it is enough to imagine that the distribution of the particles of an ideal gas by velocity, momentum of the gas molecules satisfies the normal distribution of the random variables with the center $\mu_{o}$ and dispersion $\sigma^{2}$. Suppose also that it is represented not in terms of the absolute values of the coordinates, as usual, but in terms of the normal coordinates normalized with a linear conversion of $y=\left(x-\mu_{o}\right) / \sigma$ to the standard form with the center $M_{y}=0$ and $\sigma=1$.

Then the employment of the statistical integral of the normal standardized (normal coordinates) distribution

$$
Z_{1}=\int_{-\infty}^{\infty} \exp \left(-\frac{y^{2}}{2}\right) \mathrm{d} y=(2 \pi)^{1 / 2}
$$

brings about the derivation of the thermodynamic functions of the form

$$
F_{1}=-k T N \ln Z_{1}=-P V, S_{1}=C_{p},
$$

correlating with Equation (6).

Whereas in using the statistical integrals of the normal distribution with absolute coordinates

$$
Z_{2}=\int_{-\infty}^{\infty} \exp \left(-\frac{x^{2}}{2 \sigma^{2}}\right) \mathrm{d} y=(2 \pi)^{1 / 2} \sigma
$$

the thermodynamic functions have the known form

$$
F_{2}=-k T N \ln Z_{2}=-P V-k T N \ln \sigma, S_{2}=C_{p}+k N \ln \sigma, \mu=k N \ln \sigma
$$

analogous to Equation (7).

Although nowadays the question as to the dependence of the statistical functions on the choice of the scaling factor and coordinate systems still remains open, the employment of the statistical integral in the form $Z_{1}$ as compared to $Z_{2}$ is more preferential. Specifically, in this instance the Equation (26)of state

$$
T S=U+P V
$$

becomes common to both the classical systems and the quantum ones. For example, this Equation (26) is applicable to the degenerate boson gas, for which

$$
S=(5 / 3) A V T^{3 / 2}, U=A V T^{5 / 2} \text { and } P=(2 / 3) A T^{5 / 2} \text {, when } A=\text { const, }
$$

and to the equilibrium photon gas [7], for which

$$
S=(4 / 3) \sigma_{o} V T^{3}, U=\sigma_{o} V T^{4} \text { and } P=(1 / 3) \sigma_{o} V T^{4} \text {, when } \sigma_{o}=\text { const } .
$$

Besides, in this case the thermodynamic functions $F$ and $S$ (as opposed to similar known functions) become additive, what eliminates the known Gibbs paradox and the need to "improve" the classical distributions through introduction of arbitrary correction multipliers $æ=\left(N ! \hbar^{3 N}\right)^{-1}$ into them.

In that event the condition $S=C_{p} \rightarrow 0$ at $T \rightarrow 0$ required under the third law of thermodynamics is carried out too. It substitutes the previously known result $S \rightarrow-\infty$ at $T \rightarrow 0$ of classical distributions, which does not agree with experimental observations.

\section{Dependence of the Molecular Distributions on the Concentration of Particles}

At present, the dependence of the probability density of the molecular distributions (for brevity sake, molecular distributions) by velocities or momenta on the concentration of particles in the Maxwell-Boltzmann statistics is denied in principle even in the action on the system of the external force field [6].

Particularly, in the Maxwell statistics determining the distribution of molecules by projections of velocities 


$$
f\left(v_{i}\right)=\left(\frac{m}{2 \pi k T}\right)^{1 / 2} \exp \left(-\frac{m v_{i}^{2}}{2 k T}\right)
$$

or momenta

$$
f\left(p_{i}\right)=\left(\frac{1}{2 \pi m k T}\right)^{1 / 2} \exp \left(-\frac{p_{i}^{2}}{2 m k T}\right) .
$$

the concentration of particles is not taken into account, while in the Boltzmann statistics determining the distribution of molecules by coordinates in the external force field the velocities of particles are not taken into consideration

$$
f(x, y, z)=B \mathrm{e}^{-\frac{U_{p}}{k T}} .
$$

In these expressions $f\left(v_{i}\right), f\left(p_{i}\right), f(x, y, z)$ is the probability density of the distribution of particles on projections of velocities, momenta and coordinates respectively, $U_{p}(x, y, z)$ is the potential energy of the particles in the external force field, $v_{i}\left(p_{i}\right)$ is the projections of velocities (momenta) in the rectangular coordinate system, $B$ is the normalization multiplier.

However, this concept does not quite agree with the classical thermodynamics. Such a conclusion is suggested by the consideration of probable molecular distributions in the ideal gas cycle incorporating the adiabatic (1-2), isochoric (1-3) and isothermal (2-3) processes, it is represented in Figure 1.

On the one hand, in that event one may assume that the distribution curves in the points 1 and 2 are alike by virtue of the constancy of entropy $\Delta S=0$ in the adiabatic process (1-2), though it does not agree with the known dependence of distributions on the temperature in the Maxwell statistics. Then an equilibrium distribution by velocities (momenta) in the point 3 can be obtained both owing to a change of temperature in the isochoric process (1-3) occurring at constant concentration of particles $n=$ const, and due to a change of the concentra-

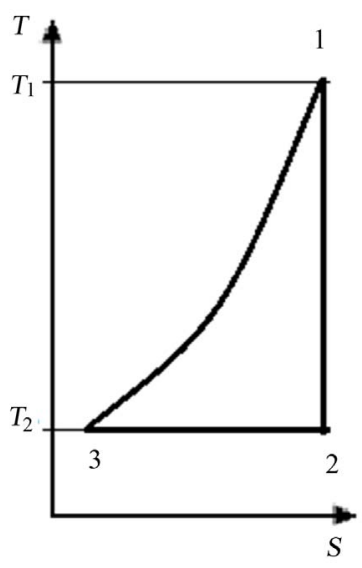

Figure 1. Ideal thermodynamic gas cycle. tion of particles in the isothermal process (2-3) occurring at constant internal energy of the system $U=$ const and in the absence (or at constancy) of the external force fields.

In this case the relationship of the probability density of the molecular distributions by the projections of velocities in the characteristic points of this cycle may be represented as curves in Figure 2 showing a possibility of obtaining the identical (equal) distribution curves both at temperature change and at concentration change.

At that, the comparison of the Maxwell distribution curves in the points 1 and 3 at temperatures $T_{1}$ and $T_{2}$ respectively (the concentration of molecules is alike $n=$ const ) is represented in Figure 2(a). In addition, the comparison of the distribution curves in the points 2 and 3 at the concentration of molecules $n_{2}$ and $n_{1}$, respectively (the temperature is alike $T_{2}=$ const) is shown in Figure 2(b).

On the other hand, to analyze the distributions in the cycle of Figure 1 one may take another assumption according to which the distribution curves in the points 1 and 2 of the adiabatic process 1-2 are diverse and correspond to a temperature change in the Maxwell statistics. However, such an assumption leading to the conclusion about the independence of the distributions from the concentration of particles should be supposedly rejected because of the contradictions regarding the determination of the statistical entropy and its association (correlation) with the molecular distributions.
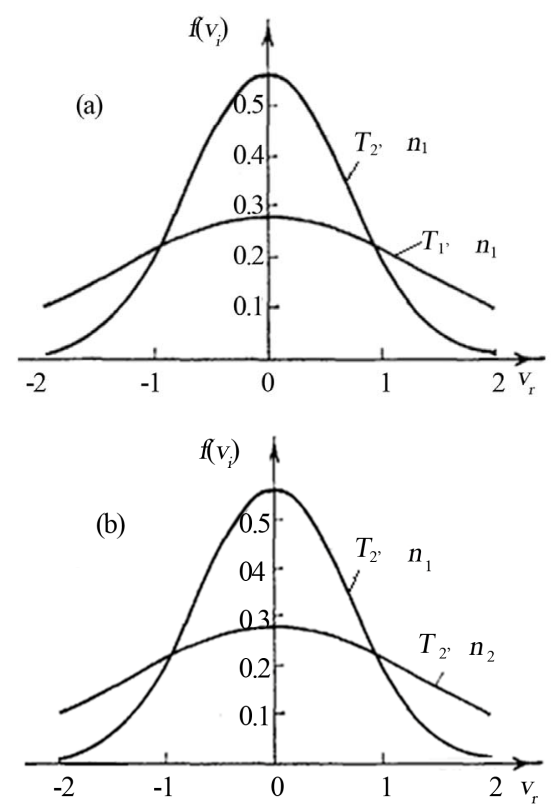

Figure 2. Comparison of the projection distributions of velocities $\left(v_{i}\right)$ in the characteristic points of the ideal cycle in Figure $1\left(v_{r}=v_{i} / \sqrt{2 k T / m}, i=x, y, z\right.$ are axes of the rectangular coordinate system). 
The problems coming about thereby cannot be cleared away completely in the Gibbs statistics too. In this statistics one considers the distribution of the molecules (to be more specific, the distribution of their representation points) in the imaginary multidimensional phase space generated by the product of the coordinates of the molecules and their momenta.

In the source prerequisites of these statistics as well as in the Maxwell-Boltzmann statistical model the principle of independence of the molecule distributions by coordinates and momenta for closed systems is adopted.

However, despite a formal denial of such dependence, it nevertheless is partially provided for, though in an implicit form, in the ultimate results obtained thereby. Thus, for example, the entropy in the Gibbs statistics, as may be seen from Equation (1), is a function of both the momenta and the concentration of particles, what is achieved owing to a correction of the primary results obtained thereby by inserting into them additional assumptions, specifically the assumption of a necessity of improving the initial distributions by application of correction multiplier æ.

Moreover, the thesis on the independence of the molecular distributions in the coordinate space (of the particle concentrations) and in the momenta space does not agree with the great canonical Gibbs distribution for the systems with a variable number of particles. In that event, the probability distribution density of particles $f(N, E)$, even in the absence of external force fields, is represented as a function

$$
f(N, E)=C \mathrm{e}^{\frac{\mu N-E}{k T}},
$$

depending both on the chemical potential $\mu$ and the number of particles in the system $N$ and on the energy of the subsystem $E=\varepsilon_{i} N_{i}$ incorporating $N_{i}$ number of particles with $\varepsilon_{i}$ energy ( $C$ is here the normalization multiplier).

However, in this case also the known statistical methods cannot explain sufficiently enough the interrelation of the entropy with the changes of the molecular distributions in the adiabatic and polytropic thermodynamic processes.

\section{Conclusion}

The performed analysis shows that some concepts of the statistical and phenomenological methods of describing the classical systems do not quite correlate with each other. Particularly, in these methods, various caloric ideal gas equations of state are employed, while the possibility of obtaining the same distributions both due to a change of concentrations and owing to a change of temperature in the thermodynamic processes is difficult to explain from the standpoint of a statistical method.
The above-mentioned difference of the equations of state may be cleared away using in the statistical functions corresponding to the canonical Gibbs equation a variable scaling factor instead of the Planck's constant. The proposed factor depends on the parameters of a system and coincides with the Planck's constant in a particular case in going of a classical system to the degenerate state. Under such an approach, the statistical entropy is transformed into one of the forms of heat capacity, what correlates with the determination of the entropy in representing normal molecular distributions with the use of the normalized coordinates.

In its turn, the agreement of the methods under consideration in the question as to the dependence of the molecular distributions on the concentration of particles, apparently, will call for further refinement of the physical model of ideal gas and the techniques for its statistical description.

In this regard, it is interesting to note that as one of the causes of difficulties arising in the harmonization of thermodynamics can be considered the deterministic approach based on the possibility of determining the coordinates and momenta of individual particles at every instant by application of the equations of the classical mechanics.

Presumably, these assumptions cannot be met enough strictly in all possible thermodynamic systems owing to a randomness of the heat motion occurring with a possibility of the initiation thereby also of the forces of collective interaction in case of a departure of the particle's parameters from their average statistical values.

To further refine the statistical conception of classical systems, the development of the statistical models based on the idea of the molecular distributions as a result of thermal fluctuations of the particle matter, i.e. on the idea of a random departure of the particles' parameters from their equilibrium values, can turn out to be more preferential.

In this instance, the functional relation between the generalized coordinates $g(x, y, z)$ and momenta $p\left(p_{x,} p_{y}, p_{z}\right)$ of the particles, which is characteristic for the known classical models, is not required, and the identical molecules of the classical systems, just as in the quantum statistics, will be considered as indistinguishable.

\section{REFERENCES}

[1] M. Kardar, "Statistical Physics of Particles," Cambridge University Press, Cambridge, 2007. doi:10.1017/CBO9780511815898

[2] I. Samkhan, Phisiks-Doklady, Vol. 41, 1996, pp. 16-18.

[3] P. W. Atkins, "Physical Chemistry," Oxford University Press, Oxford, 1978.

[4] I. Samkhan, The Open Fuels \& Energy Science Journal, 
No. 2, 2009, pp. 8-17.

http://www.benthamscience.com/open/toefj

[5] D. V. Sivukhin, "Thermodynamics and Molecular Physics,” 5th Edition, FITMAZLIT, Moscow, 2005.

[6] Yu. B. Rumer and M. Sh. Ryvkin, "Termodinamika, sta- tisticheskayafizikairinetika (Thermodynamics, Statistical Physics, and Kinetics),” Nauka, Moscow, 1977.

[7] L. D. Landau and E. M. Lifshitz, "Statistical Physics," Pergamon Press, Oxford, 1980. 